\title{
Accurate calibration of thermophotovoltaic efficiency
}

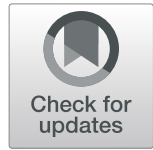

\author{
Zunaid Omair ${ }^{1}$ (D), Luis M. Pazos-Outon ${ }^{1}$, Myles A. Steiner ${ }^{2}$ and Eli Yablonovitch ${ }^{1 *}$
}

\author{
* Correspondence: eliy@eecs. \\ berkeley.edu \\ ${ }^{1}$ Department of EECS, University of \\ California, Berkeley, Berkeley, USA \\ Full list of author information is \\ available at the end of the article
}

\begin{abstract}
The new record efficiency in Thermophotovoltaics relies upon a highly reflective rear mirror. The excellent rear mirror boosts voltage by enhancing the luminescence extraction, and separately also reflects low energy photons, which would otherwise be useless in thermophotovoltaics. The reflected low energy photons reheat the thermal emitter, and regenerate above-bandgap energy photons. The efficiency calibration for such regenerative thermophotovoltaics depends on several factors, yet predominantly on the accurate measurement of the rear mirror reflectivity. Here, we report on the technique for accurate measurement of mirror reflectivity, and of record thermophotovoltaic efficiency $29.1 \pm 0.6 \%$, at $1207^{\circ} \mathrm{C}$.

Keywords: Thermophotovoltaics, Heat engine, Calibration, Energy, Photovoltaics
\end{abstract}

\section{Introduction}

Thermophotovoltaics utilizes a photovoltaic cell to generate electricity from the thermal radiation of a hot emitter [1]. We can achieve high electrical power from the thermophotovoltaic cells, requiring no moving parts. Thus, it is an attractive power source for hybrid and unmanned vehicles [2, 3], deep space probes [4-6], co-generation [7-9], concentrated solar energy [10], and compatible with thermal energy storage [11-13].

Recently we reported a record thermophotovoltaic efficiency of $29.1 \%$ at $1207^{\circ} \mathrm{C}$, by employing a highly reflective rear mirror [14]. Photons with energy smaller than the band-edge of the semiconductor do not get absorbed (Fig. 1a), and are wasted in typical thermophotovoltaic cells. The use of a highly-reflective mirror at the rear of the cell ensures that those photons can be returned back to the thermal source, thus reheating the emitter. Through this concept of photon regeneration, the thermophotovoltaic power conversion efficiency is tremendously improved. Extremely low parasitic absorption in the thermophotovoltaic cell is the most critical requirement for high thermophotovoltaic power conversion efficiency. This idea of regenerative thermophotovoltaics has further been employed to demonstrate an even higher efficiency record recently, $32 \%$ at $1182^{\circ} \mathrm{C}$ [15].

In this paper, we describe an accurate method to measure the heat to electricity conversion efficiency in regenerative thermophotovoltaics. This is based on an accurate measurement of the reflectivity of the thermophotovoltaic cell. We also use the short-

(c) The Author(s). 2020 Open Access This article is licensed under a Creative Commons Attribution 4.0 International License, which permits use, sharing, adaptation, distribution and reproduction in any medium or format, as long as you give appropriate credit to the original author(s) and the source, provide a link to the Creative Commons licence, and indicate if changes were made. The images or other third party material in this article are included in the article's Creative Commons licence, unless indicated otherwise in a credit line to the material. If material is not included in the article's Creative Commons licence and your intended use is not permitted by statutory regulation or exceeds the permitted use, you will need to obtain permission directly from the copyright holder. To view a copy of this licence, visit http://creativecommons.org/licenses/by/4.0/. 
(a)

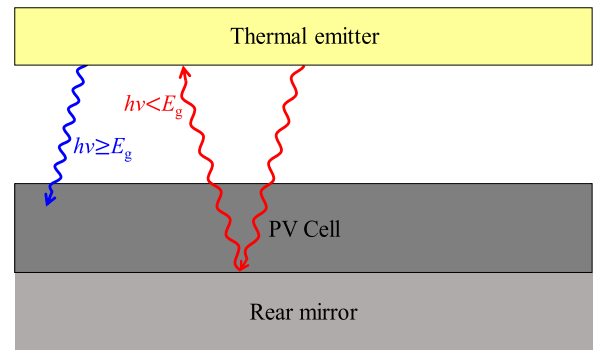

(b)

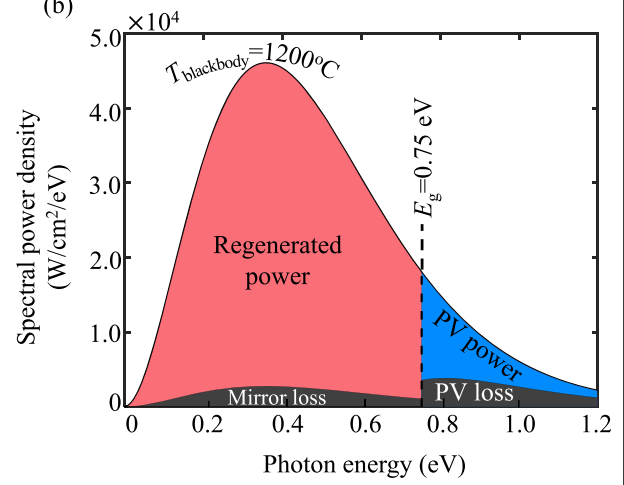

Fig. 1 Power conversion in regenerative thermophotovoltaics. Photons with energies below the band-edge of thermophotovoltaic cell are reflected by the mirror to reheat the emitter. This results in photon regeneration and a tremendous efficiency boost. Mirror inefficiency leads to below band-edge loss. Above the band-edge, losses are mostly due to defects in the cell material, Auger recombination as well as thermalization of high energy charge carriers

circuit current of the cell to calibrate the incident heat radiation precisely. This provides a very simple and sensitive calibration procedure for regenerative thermophotovoltaics. This further improves the calibration accuracy we previously reported for a record $29.1 \% \mathrm{TPV}$ efficiency, at an emitter temperature of $1207^{\circ} \mathrm{C}$.

\section{Power conversion efficiency in thermophotovoltaics}

In regenerative thermophotovoltaics, any unabsorbed portion of the radiation can be reflected by the thermophotovoltaic cell to re-generate heat at the emitter. As such, heat-to-electricity conversion efficiency at an emitter temperature $T_{\mathrm{s}}$ is defined as:

$$
\eta_{\text {thermophotovoltaic }}\left(T_{s}\right)=\frac{P_{\text {electrical }}\left(T_{s}\right)}{P_{\text {absorbed }}\left(T_{s}\right)}=\frac{P_{\text {electrical }}\left(T_{s}\right)}{\int_{0}^{\infty}\{1-R(E)\} P_{\text {incident }}(E, T s) d E}
$$

where, $P_{\text {electrical }}$ is the electrical power generated by the thermophotovoltaic cell, and $P_{\text {incident }}$ is the total optical power incident on the cell. $\mathrm{P}_{\text {absorbed }}$ is the power absorbed by the cell and $E$ is the photon energy. $R(E)$ is the spectrally-resolved reflectivity of the thermophotovoltaic cell. Calibrating the thermophotovoltaic efficiency, therefore, depends on the accurate calibration of (i) electrical power generated by the cell $\mathrm{P}_{\text {electrical }}$ $\left(\mathrm{T}_{\mathrm{s}}\right)$, (ii) thermal power incident on the cell $\mathrm{P}_{\text {incident }}\left(\mathrm{E}, \mathrm{T}_{\mathrm{s}}\right.$ ), and (iii) thermal radiation reflectivity $R(E)$ of the thermophotovoltaic cell. In the next section, we describe the methods to measure these three factors.

\section{Experimental methods}

We use a graphite ribbon as the emitter in our experiment. We accomplish the Joule heating of the emitter by passing an alternating current through the graphite thermal emitter. Beneath the emitter, the thermophotovoltaic cell is placed under an Au-coated $\mathrm{Cu}$ baffle. The baffle minimizes any stray photons from reaching the cell. Gold coating on the baffle ensures a very high $(\sim 98 \%)$ reflectivity of the stray photons. These photons would otherwise heat the baffle and the chamber through parasitic absorption. The positive and negative electrodes of the cell are wire-bonded to electrical pads for measuring the current-voltage characteristics. The thermophotovoltaic cell is kept at a 
standard temperature of $25^{\circ} \mathrm{C}$ by active water cooling. For our experiment, we used thermophotovoltaic cells with an $\operatorname{In}_{0.55} \mathrm{Ga}_{0.45} \mathrm{As}$ active layer. Details of the cell design and the growth can be found in ref. [14].

The entire setup is placed in a $10^{-5}$ Torr vacuum to minimize conductive and convective heat transfer, between the emitter and the photovoltaic cell. This also prevents oxidation of the graphite emitter at elevated temperatures.

\section{Results and discussion}

The thermophotovoltaic efficiency is the ratio of the electrical power extracted to the power absorbed by the cell. The measurement of the electrical power extracted from the thermophotovoltaic cell, $P_{\text {electrical }}$, is routine. We make wire-bonds to the cell electrodes, as shown in Fig. 2, and measure the current-voltage response.

Measurement of the absorbed power depends on the measurement of the cell reflectivity and the incident thermal radiation. Incident thermal radiation on the cell depends on three factors; (i) temperature of the emitter $\left(T_{\mathrm{s}}\right)$, (ii) emissivity $\varepsilon$ of the graphite emitter, and (iii) the geometric view factor $F$, which is the solid angle subtended by the emitter as seen from the thermophotovoltaic cell, controlled by a geometric baffle. Together with the surface area $A_{\text {cell }}$ of the thermophotovoltaic cell, the incident power is $P_{\text {incident }}\left(E, \mathrm{~T}_{\mathrm{s}}\right)=A_{\text {cell }} \varepsilon F_{\text {eff }} b_{\mathrm{s}}\left(E, T_{\mathrm{s}}\right) d E$ where, $b_{\mathrm{s}}\left(E, T_{\mathrm{s}}\right) d E=\frac{2 \pi E^{3}}{c^{2} \hbar^{3}\left(\exp \left(\frac{E}{k_{B} T}\right)-1\right)} d E$ is the black body power density in units of $\mathrm{W} / \mathrm{m}^{2}$ radiated from the emitter at temperature $T_{\mathrm{s}}$, and $\varepsilon$ is the graphite emissivity. We are introducing an effective view factor $F_{\text {eff }}$ to take into account the multiple photon bounces. The effective view factor is also dependent on the graphite emissivity owing to multiple photon reflections between the graphite emitter and the thermophotovoltaic cell. In our method of calibration detailed geometric analysis is unnecessary since $\varepsilon F_{\text {eff }}$ can be obtained directly from the observed short-circuit current.

Using the expression of $P_{\text {incident }}$, we can measure the power absorbed $P_{\text {absorbed }}$ by the thermophotovoltaic cell as:

$$
P_{\text {absorbed }}\left(T_{s}\right)=A_{\text {cell }} \mathcal{E} F_{\text {eff }} \int_{0}^{\infty}\{1-R(E)\} b_{\mathrm{s}}\left(E, T_{\mathrm{s}}\right) d E
$$

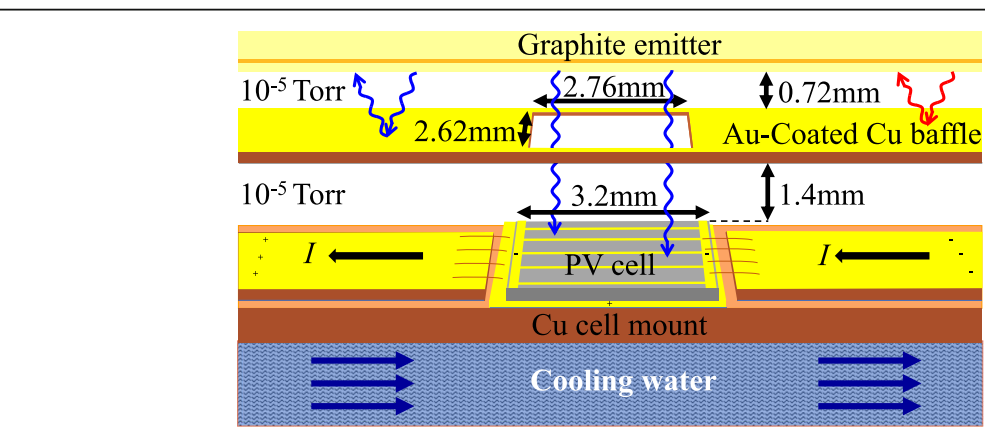

Fig. 2 Experimental setup for the thermophotovoltaic efficiency calibration. The graphite emitter is heated by Joule heating. The cell sits under a baffle, to minimize any stray photons reaching the cell. The wire bonds are made separately, to the top and bottom electrodes, for electrons and holes respectively. The cell is cooled to a standard temperature, $25^{\circ} \mathrm{C}$. Current ( () and voltage are measured by wire-bonding the cell to electrical pads 
Note that we are ignoring the photon energy dependence of the emissivity, and its temperature dependence, based on the experimental evidence that such dependency is very weak [16]. We now describe the procedure to calibrate the emitter temperature $T_{\mathrm{s}}$, and the power absorbed $P_{\text {absorbed }}\left(T_{\mathrm{s}}\right)$ by the thermophotovoltaic cell. These can be calibrated directly from the short-circuit current of the thermophotovoltaic cell.

Under illumination from a thermal emitter at temperature $T_{\mathrm{s}}$, we can express the short-circuit current as:

$$
J_{\mathrm{sc}}\left(T_{\mathrm{s}}\right)=q A_{\mathrm{cell}} \varepsilon F_{\mathrm{eff}} \int_{0}^{\infty} E Q E(E) \cdot \frac{b s(E, T s)}{E} d E
$$

We can isolate $\varepsilon F_{\text {eff }}$ on the left as:

$$
\varepsilon F_{\text {eff }}=\frac{J_{\text {sc }}\left(T_{\mathrm{s}}\right)}{q A_{\text {cell }} \int_{0}^{\infty} E Q E(E) \cdot \frac{b s(E, T s)}{E} d E}
$$

where $q$ is the electron charge and the external quantum efficiency, and $E Q E$ is the probability that a photon incident on the photovoltaic cell generates an electron-hole pair and is electrically extracted. We can write $E Q E$ as $C \times(1-R)$, where $C$ is the internal quantum efficiency of the thermophotovoltaic cell. $C$ is zero below the bandgap. We can replace $E Q E$ with $C \times(1-R)$ and change from 0 to $E_{\mathrm{g}}$ the lower limit of the denominator integral:

$$
\varepsilon F_{\text {eff }}=\frac{J_{\mathrm{sc}}\left(T_{\mathrm{s}}\right)}{q A_{\text {cell }} C \int_{E_{\mathrm{g}}}^{\infty}\{1-R(E)\} \cdot \frac{b s(E, T s)}{E} d E}
$$

where we have pulled $C$ out of the integral. We assume spectrally-averaged $C$ to be relatively constant.

We now discuss our procedure for measuring $R(E)$ and $C$ to measure emitter temperature $T_{\mathrm{s}}$, using Eq. (4). Once we know the reflectivity $R(E)$ and internal quantum efficiency $C$ at emitter temperature $T_{\mathrm{s}}$, the corresponding short-circuit current $J_{\mathrm{sc}}\left(T_{\mathrm{s}}\right)$ calibrates the emissivity-view factor product $\varepsilon F_{\text {eff }}$.

We measure the spectral reflectivity $R(E)$ of the cell using a Fourier Transform Infrared (FTIR) spectrometer. The measured spectral reflectivity is shown in Fig. 3a, averaging about $94.5 \%$ sub-bandgap reflectivity. The above bandgap reflectivity of the thermophotovoltaic cell is $34.5 \%$, the Fresnel reflectivity of the front surface (this is unchanged even beyond our above bandgap measurement range, $0.75 \mathrm{eV}-1.0 \mathrm{eV})$. Thus the spectral absorptivity $\{1-R(E)\}$ in Eq. (4) is calibrated.

We calibrate the emitter to a reference temperature $T_{\mathrm{s}}=1085^{\circ} \mathrm{C}$ by placing a copper particle on top of the graphite ribbon emitter and monitor its melting point by a change in color. We then measure the reference short-circuit current and the opencircuit voltage at $T_{\mathrm{s}}=1085^{\circ} \mathrm{C}$. We can infer the average internal quantum efficiency $C$ from the measured voltage at the reference temperature $T_{\mathrm{s}}=1085^{\circ} \mathrm{C}$.

Photon absorption primarily happens in the active layer. The generated electron-hole pairs are then efficiently transported to the electrical contacts. The internal quantum efficiency of the thermophotovoltaic cell is the product of the transport efficiency, and the optical absorption fraction inside the active layer. We can calculate the transport efficiency using the familiar base transport efficiency [17] expression $T_{\mathrm{r}}=\left[1-\left(L / L_{D}\right)^{2}\right]$, 

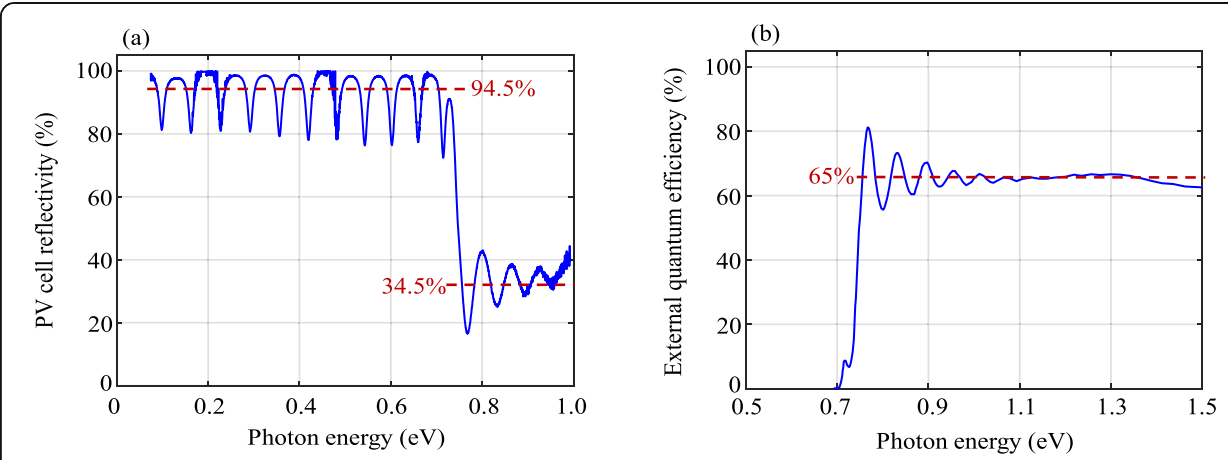

Fig. 3 Reflectivity and external quantum efficiency of one of the thermophotovoltaic cells. For this cell, subbandgap reflectivity is $94.5 \%$. The above-bandgap reflectivity is the $34.5 \%$ Fresnel reflectivity at the airsemiconductor interface. The cell converts $65 \%$ of the above-bandgap incident photons to electron-hole pairs, as given by the external quantum efficiency on the right

where $L$ is the diffusing distance, and $L_{D}$ is the diffusion length $\sqrt{ }(\mathrm{D \tau})$. In the Appendix, we show that $L_{D} \sim 19 \mu \mathrm{m}$. The active layer thickness is $2.5 \mu \mathrm{m}$. In our case, the diffusing distance $L$ is half the active layer thickness, $L=1.25 \mu \mathrm{m}$ for the following reason. The photon recycling events spread the minority carriers evenly throughout the active thickness, and so the average diffusing distance is halved. This produces a transport efficiency $T_{\mathrm{r}}=99.6 \%$, which must be multiplied by the optical absorption fraction [1$\exp \{-2 \alpha L\}]=99.3 \%$ for double-pass absorption with $\alpha=10^{4} / \mathrm{cm}$, near the band-edge. The product of $T_{\mathrm{r}}$ and the absorption fraction is $C=98.9 \%$, and we note $E Q E \equiv C \times(1-R)$.

Alternately, we measure $E Q E$ from spectrally resolved short-circuit, as shown in Fig. 3b. The internal quantum efficiency $C=99.2 \%$ estimated from spectrally resolved short circuit current is a close match to the $C=98.9 \%$ estimated from the diffusion length.

Eq. (4), combined with known graphite temperature, known short- circuit current, measured absorptivity $\{1-R\}$, and measured internal quantum efficiency calibrates the emissivity-view factor product $\varepsilon F_{\text {eff }}=0.32$. For the given emissivity-solid angle product, only $32 \%$ of the potential short circuit current was collected.

We can then use this measured $\varepsilon F_{\text {eff }}$, for calibration of emitter temperatures other than $T_{\mathrm{s}}=1085^{\circ} \mathrm{C}$, by the changed short circuit current at each temperature. At each temperature $T_{\mathrm{s}}$, we use the measured $C$ and the spectrally resolved absorptivity $\{1-R(E)\}$ to obtain the emitter temperature. The calibrated temperatures are shown in Fig. 4a.

We monitor the electrical power extracted from the thermophotovoltaic cell at the corresponding temperatures. The current-voltage response of the thermophotovoltaic cell is shown in Fig. 4b. The emitter temperature $T_{\mathrm{s}}$ and the electrical power generated $P_{\text {electrical }}\left(T_{\mathrm{s}}\right)$, are steps toward the thermophotovoltaic cell efficiency $\eta\left(T_{\mathrm{s}}\right) \equiv P_{\text {electrical }}$ $\left(T_{\mathrm{s}}\right) / P_{\text {absorbed }}\left(T_{\mathrm{s}}\right)$. We now describe the procedure for calibrating the denominator $P_{\mathrm{ab}-}$ sorbed $\left(T_{\mathrm{s}}\right)$.

We can explicitly determine $P_{\text {absorbed }}$ at emitter temperature $T_{\mathrm{s}}$, by plugging Eq. (3') into Eq. (2) as follows:

$$
P_{\text {absorbed }}\left(T_{s}\right)=\frac{J_{s c}\left(T_{s}\right) \int_{0}^{\infty}\{1-R(E)\} b s(E, T s) d E}{q \int_{0}^{\infty} E Q E(E) \cdot \frac{b s(E, T s)}{E} d E} .
$$



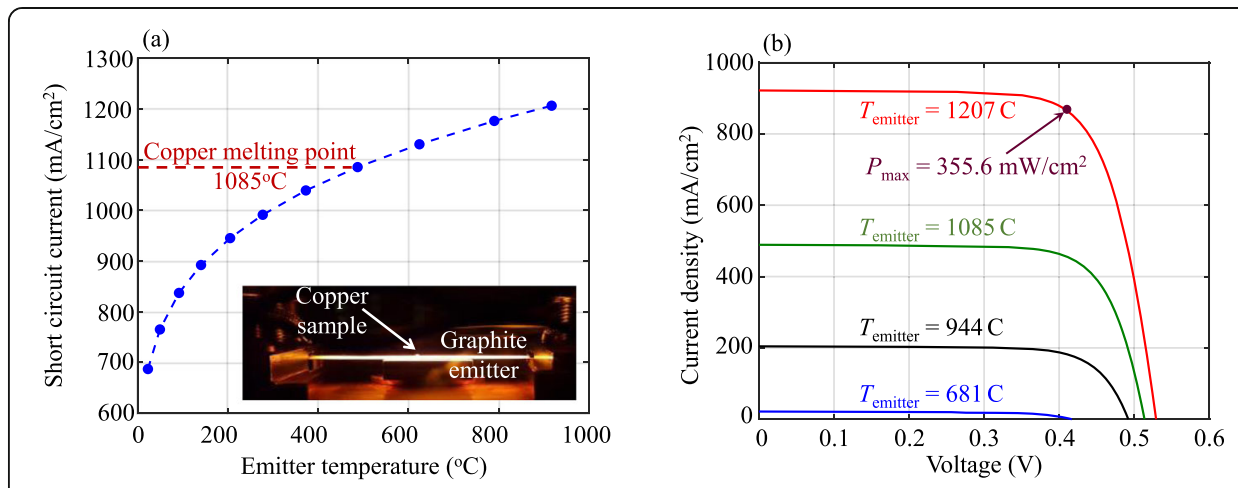

Fig. 4 Calibrating graphite emitter temperature by means of short circuit current measurement. In the first step, short-circuit current is at a reference temperature $T=1085^{\circ} \mathrm{C}$, the melting point of copper, on top of the graphite strip emitter, as shown with the inset in (a). The short circuit current in (b) calibrates temperatures at $T \neq 1085 \mathrm{C}$ from the corresponding black body spectra at each temperature

We can substitute $E Q E$ with $C \times(1-R)$ and change the lower limit on the denominator integral from 0 to $E_{\mathrm{g}}$, similar to Eq. (4):

$$
\begin{aligned}
& P_{\text {absorbed }}\left(T_{s}\right)= \frac{J_{s c}\left(T_{s}\right) \int_{0}^{\infty}\{1-R(E)\} b s(E, T s) d E}{q \int_{E_{g}}^{\infty} C\{1-R(E)\} \cdot \frac{b s(E, T s)}{E} d E} \\
&=\frac{J_{s c}\left(T_{s}\right)}{q}\left[\left(\frac{1-R_{\text {below }}}{1-R_{\text {above }}}\right) \frac{1}{C} \frac{\int_{0}^{E_{g}} b_{s}\left(E, T_{s}\right) d E}{\int_{E_{g}}^{\infty} \frac{b s(E, T s)}{E} d E}+\frac{1}{C} \frac{\int_{E_{g}}^{\infty} b_{s}\left(E, T_{s}\right) d E}{\int_{E_{g}}^{\infty} \frac{b s(E, T s)}{E} d E}\right]
\end{aligned}
$$

where, $R_{\text {below }}$ and $R_{\text {above }}$ are the spectral average reflectivities, below and above the band-edge photon energy, respectively. The reflectivities $R_{\text {below }}$ and $R_{\text {above, }}$ averaged over round-trip oscillations, are taken as constant and removed from under the integrals. We have already measured the internal quantum efficiency $C=98.9 \%$ in Eq. (6). There are three black-body integrals in Eq. (6) are also exactly known since the temperature $T_{\mathrm{s}}$ is accurately calibrated. The power conversion efficiency, Eq. (1), is a ratio of useful electrical power to the total thermal power absorbed by the thermophotovoltaic cell. Those quantities are shown in Fig. 5a. The corresponding efficiency is in Fig. 5b. We obtain a record $29.1 \pm 0.6 \%$ efficiency at $1207^{\circ} \mathrm{C}$. We now describe the procedure of error measurement in our thermophotovoltaic experiment.

\section{Error analysis}

In this section, we measure the accuracy of the thermophotovoltaic efficiency calibration using the cell reflectivity, internal quantum efficiency, and short-circuit current. The accuracy of this method depends largely on temperature calibration using the melting point of copper and then convolving the corresponding black-body spectrum with the measured short circuit current. The reduction in short circuit current relative to the full black-body spectrum is accounted for by the emissivity-solid angle factor, $\varepsilon F_{\text {eff. }}$ We obtain this emissivity-solid angle factor by measuring the short-circuit current and the internal quantum efficiency. 

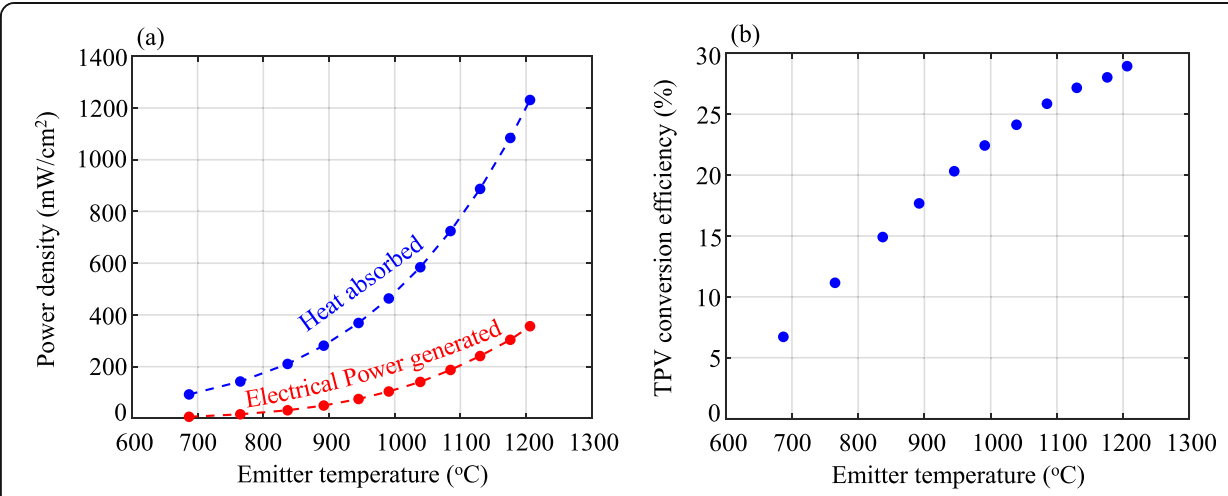

Fig. 5 Record thermophotovoltaic efficiency. Measured total incident thermal radiation absorbed, and the maximum electrical power generated by the thermophotovoltaic cell at those emitter temperatures, are shown in (a). Power conversion efficiency, the fraction of the absorbed heat that gets converted to the electrical power, is given in (b)

The thermophotovoltaic efficiency is the ratio of electrical power generated by the thermophotovoltaic cell, to the thermal power absorbed by the cell. We measure the electrical power generated by the thermophotovoltaic cell very precisely with a Keithley 2400 source meter (with nanovolt precision). As such, the error in our efficiency measurement is entirely due to the error in the estimation of $P_{\text {absorbed }}$ with $\delta \eta / \eta=\mid \delta P_{\text {ab- }}$ sorbed $/ P_{\text {absorbed }} \mid$.

The absorbed power at emitter temperature $T_{\mathrm{s}}$ depends on the Planck spectrum $b_{\mathrm{s}}(E$, $T_{\mathrm{S}}$ ), the spectral absorptivity $\{1-R(E)\}$, and the internal quantum efficiency $C$, as shown in Eq. (6). We now show how each of these physical parameters affects the accuracy of thermophotovoltaic efficiency measurement.

The incident power is determined from black body radiation at the reference temperature $T_{\mathrm{s}}=1085^{\circ} \mathrm{C}$, the melting point of copper. We use a very slow temperature ramp $\left(20^{\circ} \mathrm{C} / \mathrm{min}\right)$ while increasing the emitter temperature from $25^{\circ} \mathrm{C}$ to $1085^{\circ} \mathrm{C}$, during the calibration of $\varepsilon F_{\text {eff. }}$ This ensures an accurate determination of $\varepsilon F_{\text {eff }}=0.32$. A faster ramp can overshoot the emitter temperature beyond the melting point of copper, and reduce the accuracy of the method. We further confirm the solid-angle subtended $F_{\text {eff }}$ by geometric modeling of the view factor. Similarly, we compare the measured emissivity $\varepsilon=0.90$ against the reported value of graphite emissivity $\varepsilon=0.91$ [16] and find a close match.

Once the temperature is well-defined using the short-circuit current, we can know the Planck spectrum $b_{\mathrm{s}}\left(E, T_{\mathrm{s}}\right)$ accurately. The uncertainty in measuring $P_{\text {absorbed }}$ then depends entirely on the accuracy of measuring $R_{\text {below }}, R_{\text {above, }}$ and $C$. Taking partial derivatives with respect to each of these three variables in Eq. (6) and then normalizing by $P_{\text {absorbed }}$, we obtain (derivation in appendix):

$$
\frac{\partial \eta}{\eta}=\frac{\partial P_{\text {absorbed }}}{P_{\text {absorbed }}}=\sqrt{\left(\left|\frac{\partial R}{1-R_{\text {below }}}\right|^{2}+\left|\frac{\partial R}{1-R_{\text {above }}}\right|^{2}\right) f_{\text {abs,below }}^{2}+\left|\frac{\partial C}{C}\right|^{2}}
$$

where, $f_{\text {abs, below }}$ represents the sub-bandgap fraction of the absorbed power. We measure the spectral reflectivity above and below the band-edge with the same spectrometer. As such, we can assume $\delta R_{\text {above }}=\delta R_{\text {below }}=\delta R$, the systematic error in the reflectivity 
measurement. The emitter temperature being accurately calibrated from the short-

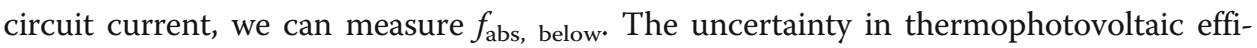
ciency $\delta \eta / \eta$ can be measured from the uncertainty of measured reflectivity $\delta R$ and the internal quantum efficiency $\delta C$.

The rear reflectivity $R$ of a regenerative thermophotovoltaic cell with an excellent rear mirror is $\geq 94 \%$. This results in a very small absorptivity $a \equiv(1-\mathrm{R})$ of the cell. For an accurate measurement of thermophotovoltaic efficiency, we need the relative uncertainty $\delta a / a=\delta R /(1-R)$ to be small. Thus the $\delta R$ of the reflectivity measurement needs to be very small. We monitored the reflectivity of a reference gold sample (NIST SRM\#1928) to be $98 \pm 0.2 \%$, in our FTIR infrared spectrometer. By averaging 200 successive scans, the remaining $\pm 0.2 \%$ error is thought to be a systematic error in the instrument rather than a statistical error.

An additional contribution to $\delta R$ is due to scattering from the electrical grid lines, on top of the thermophotovoltaic cells, as shown in Fig. 2. The electrical grid lines on the top of the thermophotovoltaic cell are 5um wide and $200 \mu \mathrm{m}$ apart. We measure the reflectivity with a $150 \mu \mathrm{m}$ spot size, in between the grid lines. The reflectivity on the Au grid-lines is $98 \%$, but at the air-semiconductor interface, the net reflectivity is $94.5 \%$. From linear interpolation, the front surface reflectivity can be $94.6 \%$, including the grids. We make this interpolation taking $2.5 \%$ front surface coverage by the gridlines into account. As such, our measured reflectivity has an additional uncertainty of $0.1 \%$. This results in net reflectivity uncertainty $98 \pm 0.3 \%$.

We can measure the errors in the internal quantum efficiency $\delta C$. We obtain $C=$ 98.9\% from the measured open-circuit voltage, as discussed in the appendix. Given the $50 \%$ uncertainty in the diffusion length, the uncertainty on our internal quantum efficiency is $98.9 \pm 0.9 \%$.

Now using the measured $\delta C$ and $\delta R$ we can calculate the efficiency uncertainty $\delta \eta$ given by Eq. (7). The relative uncertainty $\delta \eta / \eta$ as a function of emitter temperature is shown in Fig. 6. As the emitter temperature increases, more photons are emitted above the band-edge than below. The error given by $\delta R \times f_{\text {abs, below }}$ decreases, while the signal $1-R_{\text {above }}$ increases with increasing emitter temperature. Therefore, the signal to noise ratio improves with temperature. This is shown in Fig. 6. At $1207^{\circ} \mathrm{C}$, the calibrated thermophotovoltaic efficiency is $29.1 \%$, with an uncertainty $29.1 \pm 0.6 \%$. In our previous results [14], the uncertainty was $29.1 \pm 0.4 \%$. However, the previous calibration depended on the calibration of emitter temperature using short-circuit current and external quantum efficiency and then convolving the Planck spectrum with the thermophotovoltaic cell absorptivity. Using a different approach based on the carrier collection efficiency, we arrive at the same efficiency, without relying on a separate measurement of external quantum efficiency.

We can compare the accuracy of our method to the traditional calorimetric method [11]. Precise calorimetry requires complete minimization of parasitic heat absorption to achieve any reasonable accuracy, which can be challenging. In our approach, we rely on the electrical measurement of short-circuit current and open-circuit voltage, and optical measurement of reflectivity. All of these measurements are routine. Thus we can potentially achieve better accuracy compared to calorimetric measurement of thermophotovoltaic cell efficiency. 


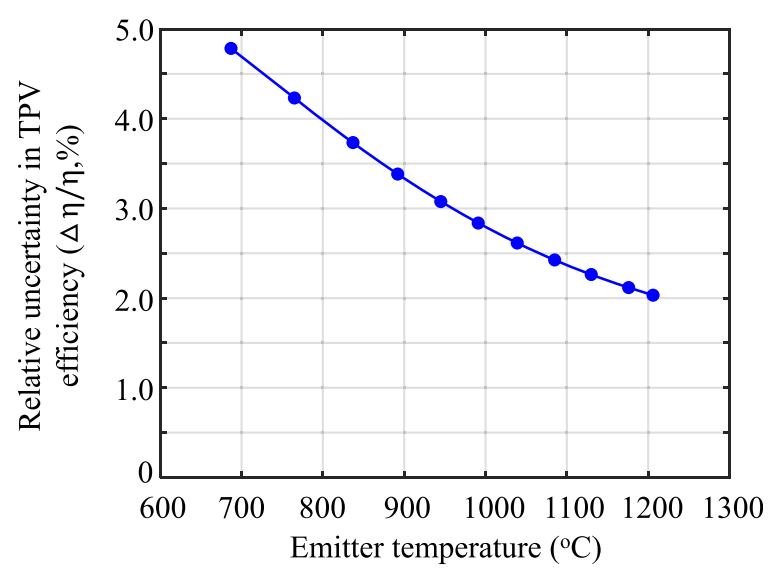

Fig. 6 Accurate calibration of record thermophotovoltaic efficiency. The uncertainty in efficiency is mainly determined by the relative uncertainty of the sub-bandgap reflectivity. As the emitter temperature increases, more photons are emitted above the band-edge than below. This results in a higher signal-tonoise ratio with increasing temperature

The thermophotovoltaic cells were scanned 200 times to reduce the corresponding random error in sub-bandgap reflectivity. The efficiency calibration using the copper melting point for temperature, and then convolving the corresponding black-body spectrum with the measured short-circuit current, provides a simple and precise absolute calibration method in thermophotovoltaics.

\section{Conclusion}

We conclude that thermophotovoltaic efficiency can be accurately calibrated through a simple procedure. The melting point of copper calibrates the absolute source temperature, and the short-circuit current of the thermophotovoltaic cell calibrates the relative source temperature. The geometric solid angle factors and the source emissivity thus become known. With a precise measurement of the sub-bandgap reflectivity of the cell, we can accurately determine the heat to the electricity conversion efficiency for the regenerative thermophotovoltaic case, in which sub-bandgap radiation is recycled to the heat source. In the regenerative case, the need for accuracy in the subbandgap reflectivity is quite critical. We improved that reflectivity measurement by repeated scans to diminish the statistical errors. Our method calibrates an efficiency of $29.1 \%$ at $1207^{\circ} \mathrm{C}$, with a corresponding error $29.1 \pm 0.6 \%$.

\section{Appendix}

\section{Minority Carrier Diffusion Length}

We have done a careful calibration of the internal photo-luminescence efficiency in the presence of photon recycling, taking mirror reflectivity into account. As in many modern photovoltaic cells, the internal photoluminescence efficiencies in our thermophotovoltaic cells are quite high, 85\% [14]. This is not as good as $~ 96 \%$ photo-luminescence efficiency of the record-breaking GaAs cells [18]. The luminescent photons are absorbed and re-emitted many times. Thus the actual radiative minority carrier lifetime is $1 /(1-0.85) \sim 6.7 \times$ longer than in the case of the lifetime in optically absorbing surroundings. 
To determine the internal quantum efficiency $C$, we need the diffusion length $\sqrt{ }(D \tau)$ which contains the carrier lifetime $\tau \equiv 1 /(B N)$ where $B$ is the bimolecular radiative recombination co-efficient, and $N$ is the majority carrier doping. But then we need to multiply that lifetime by 6.7 , for photon recycling. We used an active n-layer doping $N=10^{17} \mathrm{~cm}^{-3}$. The minority hole diffusion constant in the $\mathrm{n}$-type InGaAs active layer is $D=7.5 \mathrm{~cm}^{2} \mathrm{~s}^{-1}$, from [19]. This value was measured in the lattice-scattering dominated regime of mobility. Ahrenkiel et al. reported a radiative recombination coefficient $B=1.43 \times 10^{-10} \mathrm{~cm}^{3} \mathrm{~s}^{-1}$ [20] for an air- $\mathrm{In}_{0.53} \mathrm{Ga}_{0.47} \mathrm{As}-\mathrm{InP}$ structure. Therefore $\tau \sim 70$ nsec. Accounting for photon recycling by multiplying by 6.7 , the radiative minority lifetime becomes $\tau \sim 470 \mathrm{nsec}$. The non-radiative in these high-quality materials is even longer $\tau \sim 2 \mu$ sec.

This gives us a minority carrier diffusion length $=\sqrt{D \tau} \sim 19 \mu \mathrm{m}$.

\section{Mathematics of Error Estimation}

The electrical power was measured very accurately with a nano voltmeter. As such, the error in our efficiency measurement is solely due to the error in the estimation of $P_{\mathrm{ab}-}$ sorbed, $\delta \eta / \eta=\left|\delta P_{\text {absorbed }} / P_{\text {absorbed }}\right|$.

We can substitute $\varepsilon F_{\text {eff }}$ in Eq. (2) from Eq. (3') as follows:

$$
P_{\text {absorbed }}=\frac{J_{s c}\left(T_{s}\right)}{q} \times \frac{\int_{0}^{\infty}\{1-R(E)\} b_{s}\left(E, T_{s}\right) d E}{\int_{0}^{\infty}\{1-R(E)\} C(E) b_{s}\left(E, T_{s}\right) / E \cdot d E}
$$

We can write $E Q E=C \times\{1-R(E)\}$, where internal quantum efficiency $C$ expresses the probability of an absorbed photon to generate electron-hole pair. $C$ is 0 below the bandgap $E_{\mathrm{g}}$. Separating the super-bandgap and sub-bandgap parts of the integral in Eq. (8), we get,

$$
\begin{aligned}
P_{\text {absorbed }}= & \frac{J_{s c}\left(T_{s}\right)}{q} \times \frac{\int_{0}^{E_{g}}\{1-R(E)\} b_{s}\left(E, T_{s}\right) d E}{\int_{E_{g}}^{\infty}\{1-R(E)\} C(E) b_{s}\left(E, T_{s}\right) / E \cdot d E}+\frac{J_{s c}\left(T_{s}\right)}{q} \\
& \times \frac{\int_{E_{g}}^{\infty}\{1-R(E)\} b_{s}\left(E, T_{s}\right) E d E}{\int_{E_{g}}^{\infty}\{1-R(E)\} C(E) b_{s}\left(E, T_{s}\right) / E \cdot d E}
\end{aligned}
$$

At a given temperature, $b_{\mathrm{s}}\left(E, T_{\mathrm{s}}\right)$ and $J_{\mathrm{sc}}\left(T_{\mathrm{s}}\right)$ are precisely known. For the ease of derivations, let's define $R_{\text {below }}, R_{\text {above }}, \dot{C}$ to denote the sub-bandgap reflectivity, superbandgap reflectivity, and the internal quantum efficiency respectively, all the terms being spectrally averaged within the limits of the respective integral. We can now re-write Eq. (9) as: 


$$
\begin{aligned}
P_{\text {absorbed }}= & \frac{J_{s c}\left(T_{s}\right)}{q} \times \frac{\left(1-R_{\text {below }}\right) \int_{0}^{E_{g}} b_{s}\left(E, T_{s}\right) d E}{\left(1-R_{\text {above }}\right) C^{\prime} \int_{E_{g}}^{\infty} b_{s}\left(E, T_{s}\right) / E \cdot d E}+\frac{J_{s c}\left(T_{s}\right)}{q} \\
& \times \frac{\left(1-R_{\text {above }}\right) \int_{E_{g}}^{\infty} b_{s}\left(E, T_{s}\right) d E}{\left(1-R_{\text {above }}\right) C^{\prime} \int_{E_{g}}^{\infty} b_{s}\left(E, T_{s}\right) / E \cdot d E} \\
= & \frac{J_{s c}\left(T_{s}\right)}{q} \times \frac{\left(1-R_{\text {below }}\right) \int_{0}^{E_{g}} b_{s}\left(E, T_{s}\right) d E}{\left(1-R_{\text {above }}\right) C^{\prime} \int_{E_{g}}^{\infty} b_{s}\left(E, T_{s}\right) / E \cdot d E}+\frac{J_{s c}\left(T_{s}\right)}{q} \\
& \times \frac{\int_{E_{g}}^{\infty} b_{s}\left(E, T_{s}\right) d E}{C^{\prime} \int_{E_{g}}^{\infty} b_{s}\left(E, T_{s}\right) / E \cdot d E}
\end{aligned}
$$

Now, we can take partial derivatives with respect to $R_{\text {below }}, R_{\text {above }}, C$, and $P_{\text {absorbed }}$ on both sides of Eq. (10). Before we do that, let us call the first term on the RHS of Eq. (10) as $A$ and the second term as $\dot{B}$. As such, $P_{\text {absorbed }}=A+\dot{B}$. Now, taking the derivatives on both sides of Eq. (10), we get,

$$
\begin{aligned}
\partial P_{\text {absorbed }}= & -\frac{\partial R_{\text {below }}}{\left(1-R_{\text {below }}\right)}\left(P_{\text {absorbed }}-\dot{\mathrm{B}}\right)+\frac{\partial R_{\text {above }}}{\left(1-R_{\text {above })}\right.}\left(P_{\text {absorbed }}-\dot{\mathrm{B}}\right) \\
& -\frac{\partial \mathrm{C}^{\prime}}{\mathrm{C}^{\prime}}\left(P_{\text {absorbed }}-\dot{\mathrm{B}}\right)-\frac{\partial \mathrm{C}^{\prime}}{\mathrm{C}^{\prime}}\left(P_{\text {absorbed }}-A\right) \\
= & -\frac{\partial R_{\text {below }}}{\left(1-R_{\text {below }}\right)}\left(P_{\text {absorbed }}-\dot{\mathrm{B}}\right)+\frac{\partial R_{\text {above }}}{\left(1-R_{\text {above }}\right)}\left(P_{\text {absorbed }}-\dot{\mathrm{B}}\right) \\
& -\frac{\partial \mathrm{C}^{\prime}}{\mathrm{C}^{\prime}}\left(2 \times P_{\text {absorbed }}-\dot{\mathrm{B}}-A\right) \\
= & -\frac{\partial R_{\text {below }}}{\left(1-R_{\text {below }}\right)}\left(P_{\text {absorbed }}-\dot{\mathrm{B}}\right)+\frac{\partial R_{\text {above }}}{\left(1-R_{\text {above }}\right)}\left(P_{\text {absorbed }}-\dot{\mathrm{B}}\right)-\frac{\partial \mathrm{C}^{\prime}}{\mathrm{C}^{\prime} P_{\text {absorbed }}}
\end{aligned}
$$

As such, the root-mean-square error is,

$$
\begin{aligned}
\partial P_{\text {absorbed }} & =\sqrt{\left|\frac{\partial R_{\text {below }}}{\left(1-R_{\text {below }}\right)}\left(P_{\text {absorbed }}-\dot{\mathrm{B}}\right)\right|^{2}+\left|\frac{\partial R_{\text {above }}}{\left(1-R_{\text {above }}\right)}\left(P_{\text {absorbed }}-\dot{\mathrm{B}}\right)\right|^{2}+\left|\frac{\partial \mathrm{C}^{\prime}}{\mathrm{C}^{\prime} P_{\text {absorbed }}}\right|^{2}} \\
& =\sqrt{\left|\frac{\partial R_{\text {below }}}{\left(1-R_{\text {below }}\right)}\right|^{2}\left(P_{\text {absorbed }}-\dot{\mathrm{B}}\right)^{2}+\left|\frac{\partial R_{\text {above }}}{\left(1-R_{\text {above }}\right)}\right|^{2}\left(P_{\text {absorbed }}-\dot{\mathrm{B}}\right)^{2}+\left|\frac{\partial \mathrm{C}^{\prime}}{\mathrm{C}^{\prime} P_{\text {absorbed }}}\right|^{2}} \\
& =\sqrt{\left|\frac{\partial R}{\alpha_{\text {below }}}\right|^{2}\left(P_{\text {absorbed }}-\dot{\mathrm{B}}\right)^{2}+\left|\frac{\partial R}{P_{\text {abbove }}}\right|^{2}\left(P_{\text {absorbed }}-\dot{\mathrm{B}}\right)^{2}+\left|\frac{\partial C}{C} P_{\text {absorbed }}\right|} \\
= & \sqrt{\left|\frac{\partial R}{P_{\text {absolow }}}\right|^{2}(1-B)^{2}+\left|\frac{\partial R}{\alpha_{\text {aboved }}}\right|^{2}(1-B)^{2}+\left|\frac{\partial C}{C}\right|^{2}} \\
\frac{\partial \eta}{\eta} & =\sqrt{\left(\left|\frac{\partial R}{\alpha_{\text {below }}}\right|^{2}+\left|\frac{\partial R}{\alpha_{\text {above }}}\right|^{2}\right) f_{\text {abs,below }}{ }^{2}+\left|\frac{\partial C}{C}\right|^{2}}
\end{aligned}
$$


This gives us the expression of relative error of the absorbed power, and in turn the relative error in thermophotovoltaic efficiency. Here, we use $\delta R_{\text {below }}=\delta R_{\text {above }}=\delta R$, the systematic error in our FTIR measurement. Also, $a$ denotes the absorptivity, with the subscript indicating the relevant part of the spectrum. From the previous section, we know $C=\hat{C}$, as quantum efficiency does not vary with the emitter temperature for our experiment. Finally, we use $B=/ P_{\text {absorbed }}=1-f_{\text {abs, below. }}$ The temperature-dependent factor $f_{\text {abs, below }}$ tells us the fraction of the thermal radiation absorbed by the cell that is below the bandgap.

\section{Acknowledgements}

Not applicable.

\section{Authors' contributions}

Z.O., L.M.P.O., M.S. and E.Y. designed the experiment. Z.O. and M.S. conducted experiment. Z.O., L.M.P.O. and E.Y. analyzed data. Z.O., L.M.P.O., M.S. and E.Y. wrote the manuscript. The author(s) read and approved the final manuscript.

\section{Funding}

Experimental design, measurement, and data analysis were supported by the Department of Energy (DOE) "LightMaterial Interactions in Energy Conversion" Energy Frontier Research Center under Grant DE-SC0001293, DOE "Photonics at Thermodynamic Limit" Energy Frontier Research Center under Grant DE-SC00019140, and Kavli Energy NanoScience Institute Heising-Simons Junior Postdoctoral Fellowship of the University of California, Berkeley. This work was co-authored by the Alliance for Sustainable Energy, LLC, the manager and operator of the National Renewable Energy Laboratory for the U.S. Department of Energy (DOE) under Contract No. DE-AC36-08GO28308. The views expressed in the article do not necessarily represent the views of the DOE or the U.S. Government. The U.S. Government retains and the publisher, by accepting the article for publication, acknowledges that the U.S. Government retains a nonexclusive, paid-up, irrevocable, worldwide license to publish or reproduce the published form of this work or allow others to do so, for U.S. Government purposes.

\section{Availability of data and materials}

The data and the relevant code are available on request from the corresponding author.

\section{Competing interests}

The authors declare no competing interests.

\section{Author details}

${ }^{1}$ Department of EECS, University of California, Berkeley, Berkeley, USA. ${ }^{2}$ National Renewable Energy Laboratory, Golden, CO, USA.

Received: 13 August 2020 Accepted: 6 October 2020

Published online: 20 October 2020

\section{References}

1. Nelson RE. A brief history of thermophotovoltaic development. Semicond Sci Technol. 2003;18:S141-3.

2. Holmquist GA. TPV power source development for an unmanned undersea vehicle. AIP Conf. Proc. 1995;321:308-14.

3. Morrison O, Michael S, Edward W, William C. Use of a thermophotovoltaic generator in a hybrid electric vehicle. AlP Conf. Proc. 1999:460:488-96.

4. Wilt D, Chubb D, Wolford D, Magari P, Crowley C. Thermophotovoltaics for space power applications. AIP Conf Proc. 2007:890:335-45.

5. Schock A, Kumar V. Radioisotope thermophotovoltaic system design and its application to an illustrative space mission. AIP Conf Proc. 1995;321:139-52.

6. Teofilo VL, Choong P, Chang J, Tseng YL, Ermer S. Thermophotovoltaic energy conversion for space. J Phys Chem C. 2008;118:7841-5.

7. Butcher TA, et al. Heat transfer and thermophotovoltaic power generation in oil fired heating systems. Appl Energy. 2011;88:1543-8.

8. Fraas LM. "Economic potential for thermophotovoltaic electric power generation in the steel industry," in 2014 IEEE 40 th Photovoltaic Specialist Conference (PVSC) (Institute of Electrical and Electronics Engineers); 2014. p. 766-70.

9. Bianchi M, Ferrari C, Melino F, Peretto A. Feasibility study of a thermos-photo-voltaic system for CHP application in residential buildings. Appl Energy. 2012;97:704-13.

10. Lenert A, et al. A nanophotonic solar thermophotovoltaic device. Nat Nanotech. 2014;9:126-30.

11. T. Narayan et al., "World record demonstration of $>30 \%$ thermophotovoltaic conversion efficiency," Proc. IEEE PVSC (2020).

12. Amy C, Seyf HR, Steiner MA, Friedman DJ, Henry A. Thermal energy grid storage using multi-junction photovoltaics. Energy Environ Sci. 2019;12:334-43.

13. Datas A, Chubb DL, Veeragavan A. Steady state analysis of a storage integrated solar thermophotovoltaic system. Prog Photovoltaics. 2013;21(5):1025-39.

14. Omair Z, et al. Ultraefficient thermophotovoltaic power conversion by band-edge spectral filtering. PNAS. 2019;116(31): 15356-61. 
15. Fan D, Burger T, McSherry S, Lee B, Lenert A, Forrest SR. Near-perfect photon utilization in an air-bridge thermophotovoltaic cell. Nature. 2020;586:237.

16. Neuer G. Spectral and total emissivity measurements of highly emitting materials. Int J Thermophys. 1995;16:257-65.

17. R. F. Pierret, Ch.10 in "semiconductor device fundamentals," Pearson, 2011. The hyperbolic cosine can be extended by Taylor series to arrive at the expression in this paper.

18. Miller OD, Kurtz S, Yablonovitch E. Strong internal and external luminescence as solar cells approach the ShockleyQuiesser limit. IEEE J. Photovolt. 2012;2(3):303-11.

19. Electrical Properties of Gallium Indium Arsenide, http://www.ioffe.ru/SVA/NSM/Semicond/GalnAs/ebasic.html, online (Accessed July 2020).

20. Ahrenkiel RK, Ellingson R, Johnston $S$, Wanlass M. Recombination lifetime of $\ln _{0.53} \mathrm{Ga}_{0.47}$ As as a function of doping density. Appl Phys Lett. 1998;72:3470-2.

\section{Publisher's Note}

Springer Nature remains neutral with regard to jurisdictional claims in published maps and institutional affiliations.

Submit your manuscript to a SpringerOpen ${ }^{\odot}$ journal and benefit from:

- Convenient online submission

- Rigorous peer review

- Open access: articles freely available online

- High visibility within the field

- Retaining the copyright to your article 